\title{
Universiteit
}

Leiden

The Netherlands

\section{Entangled mixed-state generation by twin-photon scattering}

Puentes, G.; Aiello, A.; Voigt, D.; Woerdman, J.P.

\section{Citation}

Puentes, G., Aiello, A., Voigt, D., \& Woerdman, J. P. (2007). Entangled mixed-state generation by twin-photon scattering. Physical Review A, 75, 032319. doi:10.1103/PhysRevA.75.032319

Version: $\quad$ Not Applicable (or Unknown)

License: $\quad$ Leiden University Non-exclusive license

Downloaded from: https://hdl.handle.net/1887/61306

Note: To cite this publication please use the final published version (if applicable). 


\title{
Entangled mixed-state generation by twin-photon scattering
}

\author{
G. Puentes, ${ }^{1}$ A. Aiello, ${ }^{1}$ D. Voigt, ${ }^{1,2}$ and J. P. Woerdman ${ }^{1}$ \\ ${ }^{1}$ Huygens Laboratory, Leiden University, P. O. Box 9504, 2300 RA Leiden, The Netherlands \\ ${ }^{2}$ Cosine Research bv, Niels Bohrweg 11, 2333 CA Leiden, The Netherlands
}

(Received 12 May 2006; published 14 March 2007)

\begin{abstract}
We report experimental results on mixed-state generation by multiple scattering of polarization-entangled photon pairs created from parametric down-conversion. By using a large variety of scattering optical systems we have experimentally obtained entangled mixed states that lie upon and below the Werner curve in the linear entropy-tangle plane. We have also introduced a simple phenomenological model built on the analogy between classical polarization optics and quantum maps. Theoretical predictions from such a model are in full agreement with our experimental findings.
\end{abstract}

DOI: 10.1103/PhysRevA.75.032319

PACS number(s): 03.67.Mn, 42.50.Dv, 42.50.Ct, 42.65.Lm

\section{INTRODUCTION}

The study of spatial, temporal, and polarization correlations of light scattered by inhomogeneous and turbid media has a history of more than a century [1]. Due to the high complexity of scattering media only single-scattering properties are known at a microscopic level [2]. Conversely, for multiple-scattering processes the emphasis is mainly on macroscopic theoretical descriptions of the correlation phenomena [3]. In most examples of the latter [4-7] the intensity correlations of the interference pattern generated by multiple-scattered light are explained in terms of classical wave coherence. On the other hand, the recent availability of reliable single-photon sources has triggered interest in quantum correlations of multiple-scattered light [8]. Generally speaking, quantum correlations of scattered photons depend on the quantum state of the light illuminating the sample. In Ref. [8], spatial quantum correlations of scattered light were analyzed for Fock, coherent, and thermal input states.

In this paper we present experimental results on quantum polarization correlations of scattered photon pairs. In particular, we study the effect of scattering devices acting on a single photon belonging to a polarization-entangled pair. The photon pairs are initially generated by spontaneous parametric down-conversion (SPDC). The initial entanglement of the input photon pairs will in general be degraded by multiple scattering. This can be understood by noting that the scattering process distributes the initial correlations of the twin photons over the many spatial modes excited along the propagation in the medium. In the case of spatially inhomogeneous media the polarization degrees of freedom are coupled to the spatial degrees of freedom generating polarization-dependent speckle patterns. If the spatial correlations of such patterns are averaged out by multimode detection, the polarization state of the scattered photon is reduced to a mixture, and the resulting polarization entanglement of the photon pairs is degraded with respect to the initial one. A related theoretical background was elaborated in $[9,10]$.

We emphasize that the present work does not aim at describing the use of entangled photons for the characterization of different scattering media. Rather, it highlights the use of different optical properties of scattering media for entangled mixed-state generation. Specifically, we show that the coupling between polarization and spatial degrees of freedom by scattering can be used for entangled mixed-state engineering. The idea of generating mixed entangled states by coupling polarization and spatial degrees of freedom is not novel [19], but here we realize this coupling by scattering processes. We believe our implementation is of interest, since it relaxes experimental constraints compared to previous linear optics approaches that achieved similar results $[18,19]$.

This paper is structured as follows. In Sec. II we report our experiments on light scattering with entangled photons. First, we present our experimental setup and briefly describe the many different optical systems that we used as scatterers. Next, we show our experimental results. The notions of generalized Werner and sub-Werner states are introduced to illustrate these results. In Sec. III we introduce a simple phenomenological model for photon scattering that fully reproduces our experimental findings. Finally, in Sec. IV we draw our conclusions.

\section{EXPERIMENTS ON LIGHT SCATTERING WITH ENTANGLED PHOTONS}

\section{A. Experimental setup}

Our experimental setup is shown in Fig. 1. A krypton-ion laser at $413.1 \mathrm{~nm}$ pumps a 1 -mm-thick $\beta$ - $\mathrm{BaB}_{2} \mathrm{O}_{4}$ (BBO) crystal, where polarization-entangled photon pairs at wavelength $826.2 \mathrm{~nm}$ are created by SPDC in a degenerate type-II phase-matching configuration [11]. Single-mode fibers (SMFs) are used as spatial filters to assure that each photon of the initial SPDC pair travels in a single transverse mode. Spurious birefringence along the fibers is compensated by suitably oriented polarization controllers (PCs). The total retardation introduced by the fibers and walk-off effects at the BBO crystal are compensated by compensating crystals (CCs; 0.5-mm-thick BBO crystals) and half-wave plates $(\lambda / 2)$, in both signal and idler paths. In this way the initial two-photon state is prepared in the polarization singlet state $\left|\psi_{s}\right\rangle=(|H V\rangle-|V H\rangle) / \sqrt{2}$, where $H$ and $V$ are labels for the horizontal and vertical polarizations of the two photons, respectively. The experimentally prepared initial singlet state $\rho_{s}^{\text {expt }}$ has a fidelity [12] with the theoretical singlet state 


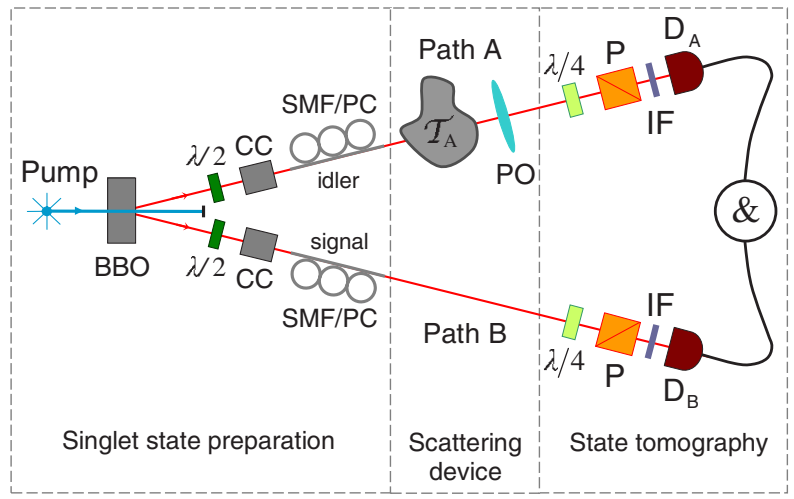

FIG. 1. (Color online) Experimental scheme: After singlet preparation, the idler photon propagates through the scattering system $\mathcal{T}_{A}$. The polarization state of the scattered photon pairs is then reconstructed via a quantum tomographic procedure (see text for details).

$\rho_{s}=\left|\psi_{s}\right\rangle\left\langle\psi_{s}\right|$ of $F\left(\rho_{s}, \rho_{s}^{\text {expt }}\right) \sim 98 \%$. In the second part of the experimental setup the idler photon passes though the scattering device $\mathcal{T}_{A}$ before being collimated by a photographic objective (PO) with focal distance $f=5 \mathrm{~cm}$. The third and last part of the experimental setup consists of two tomographic analyzers (one per photon), each made of a quarterwave plate $(\lambda / 4)$ followed by a linear polarizer $(P)$. Such analyzers permit a full tomographic reconstruction, via a maximum-likelihood technique [13], of the two-photon state. Additionally, interference filters (IFs) put in front of each detector $(\Delta \lambda=5 \mathrm{~nm})$ provide for bandwidth selection. Detectors $D_{A}$ and $D_{B}$ are "bucket" detectors, that is, they do not distinguish which spatial mode a photon comes from; thus each photon is detected in a mode-insensitive way.

\section{B. Scattering devices}

All the scattering optical systems that we used were located in the path of only one of the photons of the entangledpair (the idler one), as shown in Fig. 1. For this reason, we refer to such systems as local scatterers. Such scatterers can be grouped in three general categories according to the optical properties of the media of which they are made [14].

Type I. Purely depolarizing media, or diffusers. Such media do not affect directly the polarization state of the impinging light but change the spatial distribution of the impinging electromagnetic field.

Type II. Birefringent media, or retarders. These media introduce a polarization-dependent delay between different components of the electromagnetic field.

Type III. Dichroic media, or diattenuators. Such media introduce polarization-dependent losses for the different components of the electromagnetic field.

Type-I scattering systems produce an isotropic spread in the momentum of the impinging photons. Examples of such scattering devices are spherical-particle suspensions (such as milk or polymer microspheres), polymer and glass multimode fibers, and surface diffusers. Type-II scattering systems are made of birefringent media, which introduce an optical axis that breaks polarization isotropy. Birefringence can be classified as "material birefringence" when it is an intrinsic property of the bulk medium (for example, a birefringent wave plate), and as "topological birefringence" when it is induced by a special geometry of the system that generates polarization anisotropy, an example of a system with topological birefringence is an array of cylindrical particles. Finally, type-III scattering systems are made of dichroic media that produce polarization-dependent photon absorbtion. Examples of such devices are commonly used polarizers. A systematic characterization of all the scattering devices that we used was given in Ref. [14].

\section{Experimental results in the tangle versus linear entropy plane}

The degree of entanglement and the degree of mixedness of the scattered photon pairs can be quantified by the tangle $(T)$, namely, the concurrence squared [15], and the linear entropy $\left(S_{L}\right)$ [16]. These quantities were calculated from the $4 \times 4$ polarization two-photon density matrix $\rho$, by using $T(\rho)=\left(\max \left\{0, \sqrt{\lambda_{1}}-\sqrt{\lambda_{2}}-\sqrt{\lambda_{3}}-\sqrt{\lambda_{4}}\right\}\right)^{2}$, where $\lambda_{1} \geq \lambda_{2} \geq \lambda_{3}$ $\geq \lambda_{4} \geq 0$ are the eigenvalues of $\rho\left(\sigma_{2} \otimes \sigma_{2}\right) \rho^{*}\left(\sigma_{2} \otimes \sigma_{2}\right)$, where $\sigma_{2}=\left[\begin{array}{cc}0 & -i \\ i & 0\end{array}\right]$, and $S_{L}(\rho)=\frac{4}{3}\left[1-\operatorname{Tr}\left(\rho^{2}\right)\right]$. Figures 2(a) and 2(b) show experimental data reported on the linear entropy-tangle plane. The position of each experimental point in such plane has been calculated from a tomographically reconstructed [13] two-photon density matrix $\rho^{\text {expt }}$. The uniform gray area corresponds to nonphysical states [17]. The dashed curve that bounds the physically admissible region from above is generated by the so-called maximally entangled mixed states (MEMSs) [18,19]. The lower continuous curve is produced by the Werner states [20] of the form $\rho_{W}$ $=p \rho_{s}+[(1-p) / 4] I_{4}(0 \leq p \leq 1)$, where $I_{4}$ is the $4 \times 4$ identity matrix. Figure 2(a) shows experimental data generated by isotropic scatterers (type I). Specifically, our type-I scatterers consisted of the following categories. (i) Suspensions of milk and microspheres in distilled water, where the sample dilution was varied to obtain different points; (ii) multimode glass and polymer fibers, where the tuning parameter exploited to obtain different points was the length of the fiber (cut-back method); (iii) surface diffusers, where the full width scattering angle was used as tuning parameter. It should be noted that suspensions of milk and microspheres are dynamic media, where Brownian motion of the microparticles induces temporal fluctuations within the detection integration time [14].

In Fig. 2(a), the experimental point at the top left corner (near $T=1, S_{L}=0$ ) is generated by the unscattered initial singlet state. The net effect of scattering systems with increasing thickness is to shift the initial datum toward the bottom right corner $\left(T=0, S_{L}=1\right)$, which corresponds to a fully mixed state.

Figure 2(b) displays experimental data generated by birefringent scattering systems (type II). As an example of a system with "material birefringence" we used a pair of wedge depolarizers in cascade [21]. Different experimental points were obtained by varying the relative angle between the optical axis of the two wedges [22]. The systems with "topological birefringence" we considered consisted of two 

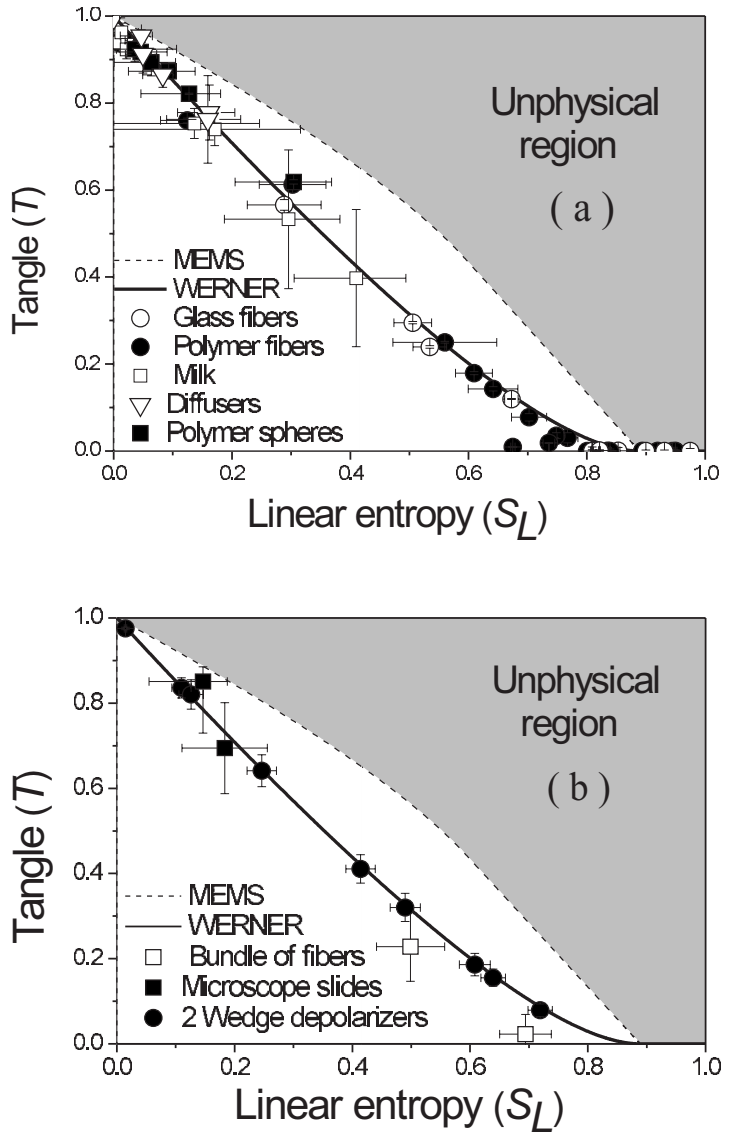

FIG. 2. Experimental data in the linear entropy-tangle $\left(S_{L}-T\right)$ plane. The gray area corresponds to unphysical density matrices. Dashed upper curve: Maximally entangled mixed states; continuous lower curve: Werner states. (a) Polarization-isotropic scatterers (type I). (b) Birefringent scatterers (type II).

different devices. (i) The first one was a bundle of parallel optical fibers [23]. Translational invariance along the fiber axes restricts the direction of the wave vectors of the scattered photons in a plane orthogonal to the common axis of the fibers. (ii) The second device was a stack of parallel microscope slides (with uncontrolled air layers in between). This optical system is depolarizing because it amplifies any initial spread in the wave vector of the impinging photon. This photon enters via a single-mode fiber (numerical aperture 0.12) from one side of the stack and travels in a plane parallel to the slides.

In Fig. 3, experimental data generated by dichroic scattering systems (type III) are shown. We used (i) surface diffusers followed by a stack of microscope slides at the Brewster angle and (ii) commercially available polaroid sheets with manually added surface roughness on its front surface to provide for wave-vector spread. All data thus obtained fall below the Werner curve, generating what we called sub-Werner states, namely, states with a lower value of tangle $(T)$ than a Werner state, for a given value of the linear entropy $\left(S_{L}\right)$.

In summary, Figs. 2(a) and 2(b) show that all data generated by type-I and -II scattering systems fall on the Werner curve, within the experimental error; while data generated by scattering samples type III, which are presented in Fig. 3, lie

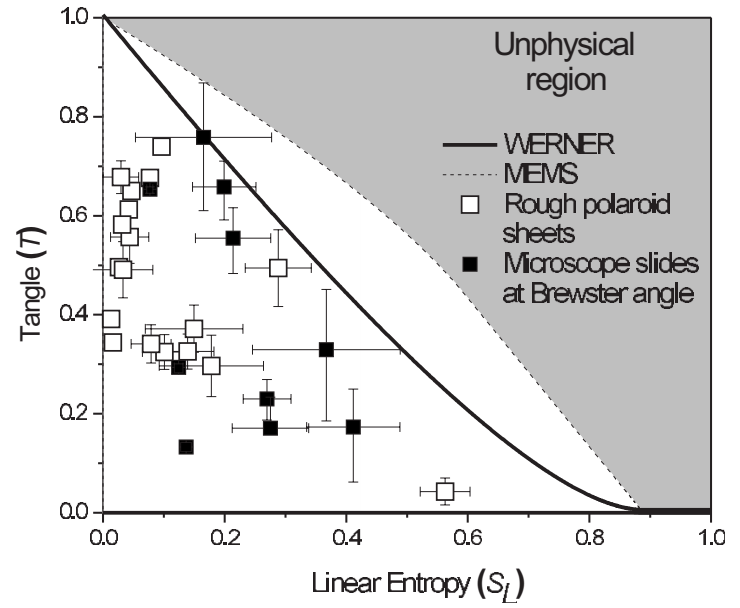

FIG. 3. Experimental data generated by dichroic scattering systems (type III).

below the Werner curve. In Sec. III we shall present a simple theoretical interpretation for such results.

\section{Error estimate}

In order to estimate the errors in our measured data, we numerically generated 16 Monte Carlo sets $N_{i}(i=1, \ldots, 16)$ of $10^{3}$ simulated photon counts, corresponding to each of the 16 actual coincidence count measurements $\left\{n_{i}^{\operatorname{expt}}\right\}$ $(i=1, \ldots, 16)$ required by tomographic analysis to reconstruct a single two-photon density matrix. Each set $N_{i}$ had a Gaussian distribution centered around the mean value $\mu_{i}=n_{i}^{\text {expt }}$, with standard deviation $\sigma_{i}=\sqrt{n_{i}^{\text {expt }} \text {. The sets } N_{i}}$ where created by using the "NormalDistribution" built-in function of the program MATHEMATICA 5.2. Once we generated the 16 Monte Carlo sets $N_{i}$, we reconstructed the corresponding $10^{3}$ density matrices using a maximum-likelihood estimation protocol, to assure that they could represent physical states. Finally, from this ensemble of matrices we calculated the average tangle $T^{\text {av }}$ and linear entropy $S_{L}^{\mathrm{av}}$.The error bars were estimated as the absolute distance between the mean quantities (av) and the measured ones (expt): $\sigma_{T}$ $=\left|T^{\text {expt }}-T^{\mathrm{av}}\right|, \sigma_{S_{L}}=\left|S_{L}^{\mathrm{expt}}-S_{L}^{\mathrm{av}}\right|$. It should be noted that this procedure produces an overestimation of the experimental errors. In the cases where part of the overestimated error bars fell into the unphysical region, the length of such bars was limited to the border of the physically allowed density matrices.

\section{E. Generalized Werner states}

Close inspection of the reconstructed density matrices generated by type-II scattering systems revealed that in some cases the measured states represented a generalized form of Werner states. These are equivalent to the original Werner states $\rho_{W}$ with respect to their values of $T$ and $S_{L}$, but the form of their density matrices is different. Werner states $\rho_{W}$ of two qubits were originally defined [20] as such states which are $U \otimes U$ invariant: $\rho_{W}=U \otimes U \rho_{W} U^{\dagger} \otimes U^{\dagger}$. Here $U \otimes U$ is any symmetric separable unitary transformation act- 
ing on the two qubits. The generalized Werner states $\rho_{G W}$ we experimentally generated, can be obtained from $\rho_{W}$ by applying a local unitary operation $V$ acting upon only one of the two qubits: $\rho_{G W}=V \otimes I \rho_{W} V^{\dagger} \otimes I$, where $I=\left[\begin{array}{cc}1 & 0 \\ 0 & 1\end{array}\right]$, and

$$
V(\alpha, \beta, \gamma)=\left[\begin{array}{cc}
e^{-(i / 2)(\alpha+\beta)} \cos \gamma / 2 & -e^{-(i / 2)(\alpha-\beta)} \sin \gamma / 2 \\
e^{(i / 2)(\alpha-\beta)} \sin \gamma / 2 & e^{(i / 2)(\alpha+\beta)} \cos \gamma / 2
\end{array}\right],
$$

where $\alpha, \beta, \gamma$ can be identified with the three Euler angles characterizing an ordinary rotation in $\mathrm{R}^{3}[24,25]$. These generalized Werner states have the same values of $T$ and $S_{L}$ as the original $\rho_{W}$ (since a local unitary transformation does not affect either the degree of entanglement or the degree of purity) but are no longer invariant under unitary transformations of the form $U \otimes U$. By using Eq. (1), we calculated the average maximal fidelity of the measured states $\rho_{G W}^{\text {expt with the }}$ target generalized Werner states $\rho_{G W}^{\text {theor }}(p, \alpha, \beta, \gamma)$. We found $\bar{F}\left(\rho_{G W}^{\text {expt }}, \rho_{G W}^{\text {theor }}\right) \approx 96 \%$, revealing that our data are well fitted by this four-parameter class of generalized Werner states.

\section{THE PHENOMENOLOGICAL MODEL}

In Ref. [27], a theoretical study of the analogies between classical linear optics and quantum maps was given. Within this theoretical framework it is possible to build a simple phenomenological model capable of explaining all our experimental results. To this end let us consider the experimental setup represented in Fig. 1. The linear optical scattering element $\mathcal{T}_{A}$ inserted across path $A$ can be classically represented by some Mueller matrix $\mathcal{M}$ [2] which describes its polarization-dependent interaction with a classical beam of light. However, $\mathcal{T}_{A}$ can also be represented by a linear, completely positive, local quantum map $\mathcal{E}: \rho \rightarrow \mathcal{E}[\rho]$, which describes the interaction of the scattering element with a twophoton light beam encoding a pair of polarization qubits. These qubits are, in turn, represented by a $4 \times 4$ density matrix $\rho$. Since $\mathcal{T}_{A}$ interacts with only one of the two photons, the map $\mathcal{E}$ is said to be local and it can be written as $\mathcal{E}=\mathcal{E}_{A}$ $\otimes \mathcal{I}$, where $\mathcal{E}_{A}$ is the single-qubit (or single-photon) quantum map representing $\mathcal{T}_{A}$, and $\mathcal{I}$ is the single-qubit identity map.

It can be shown that the classical Mueller matrix $\mathcal{M}$ and the single-qubit quantum map $\mathcal{E}_{A}$ are univocally related. Specifically, if with $\mathcal{M}$ we denote the complex-valued Mueller matrix written in the standard basis, then the following decomposition holds:

$$
\mathcal{M}=\sum_{\mu=0}^{3} \lambda_{\mu} T_{\mu} \otimes T_{\mu}^{*},
$$

where $\left\{T_{\mu}\right\}$ is a set of four $2 \times 2$ Jones matrices [2], each representing a nondepolarizing linear optical element in classical polarization optics, and $\left\{\lambda_{\mu}\right\}$ are the four non-negative eigenvalues of the "dynamical" matrix $H$ associated to $\mathcal{M}$. Given Eq. (2), it is possible to show that the two-qubit quantum map $\mathcal{E}$ can be written as
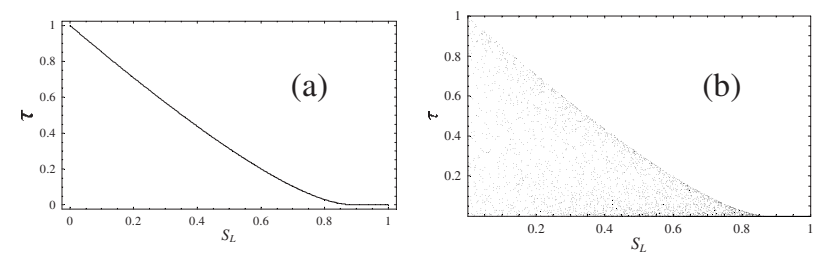

FIG. 4. Numerical simulation for our phenomenological model. (a) Isotropic and birefringent scattering; (b) dichroic scattering.

$$
\rho_{\mathcal{E}}=\mathcal{E}[\rho] \propto \sum_{\mu=0}^{3} \lambda_{\mu} T_{\mu} \otimes I \rho T_{\mu}^{\dagger} \otimes I,
$$

where the proportionality symbol on the right-hand side of Eq. (3) accounts for a possible renormalization to ensure $\operatorname{Tr}\left(\rho_{\mathcal{E}}\right)=1$. Such renormalization becomes necessary when $\mathcal{T}_{A}$ presents polarization-dependent losses (i.e., dichroism). We anticipate that when such renormalization is necessary the map is considered non-trace-preserving. We shall briefly discuss this issue in the conclusion.

With these ingredients, a phenomenological polarizationscattering model can be built as follows. First we use the polar decomposition [26] to write an arbitrary Mueller matrix $\mathcal{M}=\mathcal{M}_{\Delta} \mathcal{M}_{B} \mathcal{M}_{D}$, where $\mathcal{M}_{\Delta}, \mathcal{M}_{B}$, and $\mathcal{M}_{D}$ represent a purely depolarizing element, a birefringent (or retarder) element, and a dichroic (or diattenuator) element, respectively. Specific analytical expressions for $\mathcal{M}_{\Delta}, \mathcal{M}_{B}$, and $\mathcal{M}_{D}$ can be found in the literature [21]. Second, we use Eq. (2) to find the quantum maps corresponding to $\mathcal{M}_{\Delta}, \mathcal{M}_{B}$, and $\mathcal{M}_{D}$ and, by using such maps, we calculate the scattered two-photon state $\rho_{\mathcal{E}}$. In our experimental realizations we used isotropic scatterers $\mathcal{M}_{I S}=\mathcal{M}_{\Delta}$ with isotropic depolarization factor 0 $\leq \Delta<1$, birefringent scattering media $\mathcal{M}_{B S}$, described in terms of the product of a purely birefringent medium $\mathcal{M}_{B}$ and an isotropic depolarizer $\mathcal{M}_{\Delta}$, i.e., $\mathcal{M}_{B S}=\mathcal{M}_{B} \mathcal{M}_{\Delta}$, and, finally, dichroic scattering media $\mathcal{M}_{D S}=\mathcal{M}_{D} \mathcal{M}_{\Delta}$, which are in turn described by a product of a purely dichroic medium $\mathcal{M}_{D}$ and a purely depolarizing medium $\mathcal{M}_{\Delta}$. It should be noted that these product decompositions are not unique. Other decompositions with different orders are possible but the elements of each matrix might change, since the matrices $\mathcal{M}_{\Delta}, \mathcal{M}_{B}$, and $\mathcal{M}_{D}$ do not commute.

Filling in the above expressions with random numbers selected from suitably chosen ranges, we simulated all scattering processes occurring in our experiments. Figure 4 shows a numerical simulation of the scattered states in the tangle vs linear entropy plane, obtained with the singlet twophoton state as input state. Figure 4(a) corresponds to isotropic and birefringent scatterers, and Fig. 4(b) to dichroic scatterers. The qualitative agreement between this model and the experimental results shown in Figs. 2 and 3 is manifest.

\section{CONCLUSIONS}

In summary, we have presented experimental results on entanglement properties of scattered photon pairs for three varieties of optical scattering systems. In this way we were able to generate two distinct types of two-photon mixed 
states; namely, Werner-like and sub-Werner-like states. Moreover, we have introduced a simple phenomenological model based on the analogy between classical polarization optics and quantum mechanics of qubits, which fully reproduces our experimental findings. In the case of sub-Werner states, the phenomenological model represents a non-tracepreserving quantum map. This description might be considered controversial since a non-trace-preserving local map can in principle lead to violation of causality when it describes the evolution of a composite system made of two spatially separate subsystems [28]. However, we argue that our measured states do not violate the no-signaling condition as they are postselected by the coincidence measurement, a proce- dure that involves classical communication between the two detectors. Finally, we expect it to be possible to create states above the Werner curve (in particular MEMSs) $[18,19]$, by postselective detection when acting on a single photon [28]. Work along this line is in progress in our group.

\section{ACKNOWLEDGMENTS}

This project is part of the program of FOM and is also supported by the EU under an IST-ATESIT contract. We gratefully acknowledge M. B. van der Mark for making available the bundle of parallel fibers [23].
[1] Lord Rayleigh, Philos. Mag. 47, 375 (1899); Proc. R. Soc. London, Ser. A 79, 399 (1907).

[2] H. C. van de Hulst, Light Scattering by Small Particles (Dover, New York, 1981).

[3] M. C. W. van Rossum and T. M. Nieuwenhuizen, Rev. Mod. Phys. 71, 313 (1999).

[4] M. P. Van Albada and A. Lagendijk, Phys. Rev. Lett. 55, 2692 (1985).

[5] A. A Chabanov, M. Stoytchev, and A. Z. Genack, Nature (London) 404, 850 (2000).

[6] G. L. J. A. Rikken and B. A. van Tiggelen, Nature (London) 381, 54 (1996).

[7] F. Scheffold and G. Maret, Phys. Rev. Lett. 81, 5800 (1998); F. C. MacKintosh, I. X. Zhu, D. J. Pine, and D. A. Weitz, Phys. Rev. B 40, 9342 (1989).

[8] P. Lodahl, A. P. Mosk, and A. Lagendijk, Phys. Rev. Lett. 95, 173901 (2005).

[9] A. Aiello and J. P. Woerdman, Phys. Rev. A 70, 023808 (2004).

[10] J. L. van Velsen and C. W. J. Beenakker, Phys. Rev. A 70, 032325 (2004).

[11] P. G. Kwiat, K. Mattle, H. Weinfurter, A. Zeilinger, A. V. Sergienko, and Y. Shih, Phys. Rev. Lett. 75, 4337 (1995).

[12] R. Jozsa, J. Mod. Opt. 41, 2315 (1994). The fidelity between the target state $\rho^{\text {tar }}$ and the measured state $\rho^{\text {expt }}$ was calculated

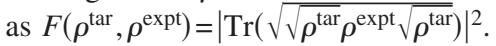

[13] D. F. V. James, P. G. Kwiat, W. J. Munro, and A. G. White, Phys. Rev. A 64, 052312 (2001).

[14] G. Puentes, D. Voigt, A. Aiello, and J. P. Woerdman, Opt. Lett.
30, 3216 (2005).

[15] W. K. Wootters, Phys. Rev. Lett. 80, 2245 (1998).

[16] S. Bose and V. Vedral, Phys. Rev. A 61, 040101(R) (2000).

[17] W. J. Munro, D. F. V. James, A. G. White, and P. G. Kwiat, Phys. Rev. A 64, 030302(R) (2001).

[18] N. A. Peters, J. B. Altepeter, D. A. Branning, E. R. Jeffrey, T.-C. Wei, and P. G. Kwiat, Phys. Rev. Lett. 92, 133601 (2004).

[19] M. Barbieri, F. De Martini, G. Di Nepi, and P. Mataloni, Phys. Rev. Lett. 92, 177901 (2004).

[20] R. F. Werner, Phys. Rev. A 40, 4277 (1989).

[21] D. S. Kliger, J. W. Lewis, and C. E. Randall, Polarized Light in Optics and Spectroscopy (Academic Press, San Diego, 1990).

[22] G. Puentes, D. Voigt, A. Aiello, and J. P. Woerdman, Opt. Lett. 31, 2057 (2006).

[23] M. B. van der Mark, Ph.D. thesis, University of Amsterdam, 1990 (unpublished).

[24] M. A. Nielsen and I. L. Chuang, Quantum Computation and Quantum Information (Cambridge University Press, Cambridge, U.K., 2000), Chap. 12.

[25] J. J. Sakurai, Modern Quantum Mechanics (Addison-Wesley, Reading, MA, 1994).

[26] S.-Y. Lu and R. A. Chipman, J. Opt. Soc. Am. A 13, 1106 (1996).

[27] A. Aiello, G. Puentes, and J. P. Woerdman, e-print quant-ph/ 0611179.

[28] A. Aiello, G. Puentes, D. Voigt, and J. P. Woerdman, e-print quant-ph/0603182. 